The meeting concluded with the thought that the WHO might provide a forum for further discussions leading to improved training in genetics at all levels.

RODNEY HARRIS

Department of Medical Genetics, St Mary's Hospital,

Hathersage Road, Manchester M13 OJH.

\section{Training course for senior registrars in clinical genetics, Brindle Lodge, NWRHA, 1 to 5 September 1986}

One of the advantages of a small specialty is that it is possible to know all the trainees personally and when we ask for their help in identifying deficiencies in training we get it promptly! Recently there was a unanimous request from trainees for information on 'Organising Genetic Services' (in preparation for their apotheosis to consultant status) and for 'Basic Genetics' (from trainees lacking a degree in genetics). There was also felt to be a need for trainees in clinical genetics to meet their counterparts in cytogenetics. A training course was planned with the help of the North Western Regional Health Authority (NWRHA), who generously made available a Regency mansion set in the Lancastrian countryside where a unique, but potentially immiscible, collection of talents was assembled. These included a National Health Service (NHS) regional general manager, a treasurer, several experts on clinical trials and health service economics, academics, clinical geneticists and cytogeneticists, and genetic and cytogenetic trainees. The outcome was indeed catalytic with considerable improvement in mutual understanding of the problems of the Health Service.

The clinical and cytogenetic trainees found each other to be agreeable companions and contact with general managers to be 'a revelation' and 'a sobering insight'. One trainee even awarded 'five stars for NHS management' (a reference to the training session on NHS management). Equally interesting was the feedback from the tutors about the trainees. The Health Service managers expressed pleasure at the interest shown and one said that he thought that geneticists were the nicest bunch of clinicians he had met!
All talks were informal with frequent interjections and extended discussion. Every speaker was allocated a trainee as 'rapporteur' who took notes, led the discussion if necessary, and clarified points with the speaker afterwards. To the surprise of one organiser, the term 'rapporteur' was unfamiliar and threatening to trainees (one even described it as "wretched") but, allowing for some initial semantic confusion, it produced a fascinating permanent record of the meeting (available from $\mathrm{RH}$ while stocks last) and avoided passive participation. This was reinforced by four 'set piece' presentations of high quality: genetic services, screening for Down's syndrome by low maternal serum AFP, Huntington's disease and the G8 probe, and finally prenatal diagnosis and future screening for cystic fibrosis. Several lectures and discussions were devoted to the clinical applications of gene probes and of microcomputers. There were four microcomputers available throughout the course allowing instruction and 'hands on' experience of a dysmorphology data base, risk, linkage, and data management programmes.

The day devoted to the aims and philosophy of NHS general management was particularly lively, matched only by the previous day's sometimes heated discussion of the complementary roles of clinical geneticists and cytogeneticists. There was much scepticism from trainees about experimental economic tools apparently already being used to make decisions on the relative merits of different clinical procedures and hence the allocation of resources. These methods included 'Quality Adjusted Life Years' (QALYs) and 'Decision Trees', neither of which appeared in their present forms to be able to accommodate genetic procedures and nondirective genetic counselling. Indeed, the existence of the latter was a surprise to the economists and it was good to have registered a palpable hit with the treasurers who, impressed by the obvious value of rapidly evolving services based on the 'New Genetics', were appalled to learn how tenuous and 'soft' its funding was. However, faced with a treasurer's dilemma of reconciling 8 million pounds worth of bids for regional developments with only 3 million in the kitty, it was clear that clinicians and scientists who can demonstrate objectively the effectiveness (and efficiency, even when discounted!) of their work will do their patients a considerable service. Most of us went away determined to think very hard about ways of proving the worth of genetics using our new armamentarium of economic jargon, although we were told that colourful brochures couched in lay terms may have the greatest impact on manager's struggling to understand the claims of competing professionals.

The present state of clinical genetics and 
